\title{
Histopathological identification of aspergillosis in animals at Dhaka Zoo
}

\author{
S. A. Ahasan ${ }^{1,2^{\star}}$, E. H. Chowdhury ${ }^{1}$, M. M. Rahman ${ }^{2}$ and M. A. Rahman ${ }^{2}$ \\ ${ }^{1}$ Department of Pathology, Bangladesh Agricultural University, Mymensingh-2202, Bangladesh and ${ }^{2}$ Department of \\ Livestock Services, Bangladesh; Krishi Khamar Sarak, Farmgate, Dhaka -1215, Bangladesh, *E-mail: \\ ahasan67@gmail.com
}

\begin{abstract}
Dhaka Zoo with 2000 animal heads of 184 species from significant genetic diversity and five million visitors' influx round the year is placing it a public health important spot. This study was conducted to investigate aspergillosis in animals at Dhaka Zoo to ascertain animal health, welfare and public health safety standard. One hundred and two necropsied tissue samples preserved in 10\% neutral buffered formalin at necropsy from 36 animals of 25 different species were collected from Dhaka Zoo. Twenty five out of 36 study animals were suffering from granulomatous diseases. Among them 13 animals were suffering from Aspergillosis. Clinical history, nodular lesions from necropsy findings, granulomatous reactions along with fungal spores and characteristic radiating club on histopathology; dichotomously branching septate hyphae and mycelial conidiophore on special staining were revealed Aspergillosis in 13 animals of nine species that includes four rhesus macaques (Macaca mulatta), two samber deer (Cervus unicolor) and one of each species were nilgai (Boselaphus tragocamelus), horse (Equus caballus), stripped hyena (Hyena hyena), gayal (Bos frontalis), beisa oryx (Oryx beisa beisa), water buck (Kobus L. leche) and greater kudu (Tragelaphus strepsiceros). Present study provides evidence of existing Aspergillosis and similar long standing zoonotic diseases in majority of rest of the animals with health risk that shades health safety standard at Dhaka Zoo.
\end{abstract}

Keywords: Granuloma, Aspergillosis, Hyphae, Zoo animals, Histopathology

\section{Introduction}

Zoo is popularly called living laboratory and knowledge generation center for wildlife implying both in-situ and ex-situ (Ahasan and Azam, 2007; Peter, 2005). Genetic, species, subspecies and population diversity shaped zoos an environmental hot spot and sourcing of disease producing microorganisms and predispose cross infection of closely related animals. Around 150 emerging and re-emerging infectious diseases are originating from or harbored in wildlife round the globe (OIE, 2000; Lisle et al., 2002) while 600 million visitor influxes each year in zoos of the whole planet (Dollinger, 2006). Considering the biological diverse range of wildlife, multiple disease susceptibility and huge visitor influx confirms zoos as an important and obligated public health concern arena (Peter, 2005; Tribe, 2004; OIE, 2000; O'Reilly and Daborn, 1995). Among others, bacterial and fungal diseases are most prevailing infections in zoo collections. The worst infectious diseases considered are mycobacteriosis and mycosis; both the groups posesses seriously zoonotic, curving the animal welfare and public health standard (WAZA, 2003) of zoos in calling it modern zoo. Even then, zoos are inseparable from studying biodiversity and environment, public health and knowledge generation center (Peter, 2005) for mankind. It is also limited with different social conflicts, health-hazards, management, behavioural menace and so forth (Salem and Rowan, 2001).

Since its inception, zoo is a potential source of plague, tuberculosis, herpes virus B (hepatitis), rabies, Marburg virus, fungus and parasitic worm among others (Renquist and Whitney, 1978; Gary et al., 2003). A recent threat has come up with West Nile and hanta viruses (Gary et al., 2003). Dhaka zoo has a prevalence of mycobacteriosis, salmonellosis, colibacillosis, coccidioidomycosis and those are diagnosed only on the basis of clinical history and necropsy records (Rahman and Ahasan, 2006 and 2007).

Aspergillosis is the name given to a wide variety of diseases caused by the fungi of the genus Aspergillus. Most humans inhale Aspergillus spores every day; Aspergillosis develops mainly in immunocompromised individuals. Typical histopathological features are infiltration of huge number of dichotomously branching septate hyphae concomitant with yeast cells, macrophages and other mononuclear cells and mycelial conidiophores indicative for aspergillosis (Jones et al., 1997). Pathogens are Aspergillus fumigatus, 
Aspergillus nidulans and Aspergillus flavus. Horse, dog (Mackinnon et al., 2000), deer, American bison at the London zoo, captive monkeys, roe-deer in Switzerland, filly (Breshears et al., 2007) were reported with aspergillosis. It was reported in alpaca (Muntz, 1999) and American bison calf (Monteros et al., 1999). Human and birds are very susceptible. Mortality is variable by host immunity and load of Aspergillus fungus.

However, these diseases were not investigated in Dhaka zoo. Therefore, the present study was undertaken to investigate the prevalence and clinico-pathological changes of Aspergillosis in animals at Dhaka zoo to apprehend public health safety standard that prevailed here.

\section{Materials and Methods}

The research work was conducted in the Department of Pathology, Bangladesh Agricultural University (BAU), Mymensingh-2202 and Dhaka Zoo, Bangladesh. A total of 102 necropsied formalin-fixed tissue specimens from 36 animals of 25 different species at necropsy were investigated clinicohistopathologically and special staining technique was followed. The study comprised of eight rhesus macaques (Macaca mulatta), four spotted deers (Cervus axis/Axis axis), two samber deers (Cervus unicolor), two golden pheasant (Chrysolophus pictus), one of each species were guineapig (Cavia porcellus), wildebeest (Connochaetes taurinus), striped hyena (Hyena hyena), Indian/Asiatic lion (Panthera leo persica), gayal (Bos frontalis), American rhea (Rhea americana), Australian terrier dog (Canis lupus familiarizes), zebra (Equus zebra hartmannae), nilgai (Boselaphus tragocamelus), horse (Equus caballus), barking deer (Muntiacus muntjak), ostrich (Struthio camelus), crested serpent-eagle (Spilorins cheela), common languor (Presbytis entellus), fishing cat (Felis viverrina), beisa oryx (Oryx beisa beisa), reticulated python (Python molurus), water buck (Kobus L. leche), greater kudu (Tragelaphus strepsiceros), and olive baboon (Papio anubis). The animal belongs to non-human primates $(n=10)$, carnivores $(n=4)$, herbivores $(n=16)$, reptiles $(n=1)$ and birds $(n=5)$.

At necropsy, tissue changes were recorded; photographed and lesioned tissues of all vital organs with special attention to nodular lesions were preserved in $10 \%$ neutral buffered formalin. Formalin fixed samples were processed for paraffin embedding, sectioning and staining as routine hematoxylin and eosin staining (Luna, 1968), periodic acid Schiff (PAS) for staining fungus according to standard method of histopathological study (Mallory, 1968). In PAS staining, 0.5\% Periodic acid solution and 0.2\% Light Green solution (Stock) were used. Briefly tissue sections were deparaffinized and hydrated to distilled water before oxidized in periodic solution for 5 minutes followed by rinsing in distilled water then dipped in Schiff reagent solution for 15 minutes. It was then washed in running tap water for 10 minutes until a pink colour to develop followed by counter staining in light green for few seconds. Again washed in running tap water for 10 minutes then dehydrated in 95\% alcohol, absolute alcohol, and cleared in xylene, two changes each followed by mounting with permount (McManus, 1948).

Photomicrograph was taken using photomicrographic camera (Olympus PM-C 35 Model) and Digital Camera Mounted Photomicrographic device (Diffrential Interference Contrast - DIC) (Olympus, Nizol FC, E-5000, 8.4V, 0.9A, CE N 150) respectively.

\section{Results and Discussion}

Out of 36 investigated animals 25 were suffering from granulomatous diseases (about 70\%, Chart 1) while Aspergillosis was found in 13 animals of four rhesus macaques (Macaca mulatta), two samber deer (Cervus unicolor); and one of each were nilgai (Boselaphus tragocamelus), horse (Equus caballus), stripped hyena (Hyena hyena), gayal (Bos frontalis), beisa oryx (Oryx beisa beisa), water buck (Kobus $L$. leche) and greater kudu (Tragelaphus strepsiceros). 


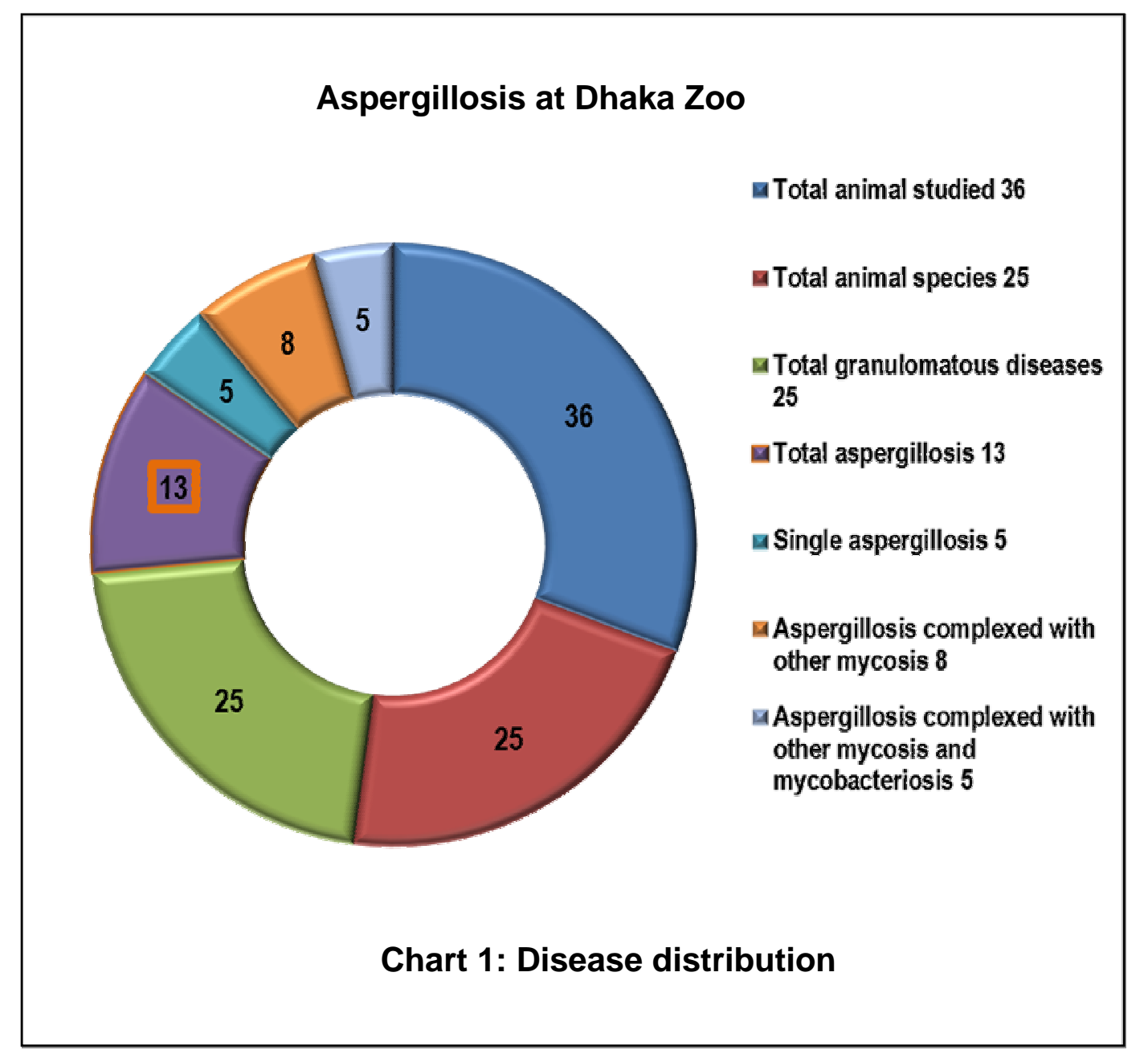

Clinically, animals that were suffering from Aspergillosis showed frequent coughing and anorexia with occasional emaciation and weakness followed by death. Necropsy unearthed findings of tiny yellow-white minute to large nodulations, cavitations, caseations, and suppurations and blackish to greenish discolourations of the affected organs (Fig. 1 to 3).

Routine histopathology depicted typical granulomatous reactions (Fig. 4) at lower magnification (10X). Multifocal to diffuse and severe forms of granuloma with formation of both Langhang's and foreign body giant cells along with fungal spores and characteristically significant radiating club in histopathological focuses were noted (Fig. 5) at higher magnification (40X, 100X). Granuloma showed limited calcification and with or without encapsulation (Fig. 4 and 5). Fungal spores and radiating club in $\mathrm{H}$ \& E staining predispose conducting special staining with PAS for fungal identification and all the cases positive in $\mathrm{H}$ \& E corresponded by PAS. Fungal radiating aggregates (clubs) and spores (Fig. 5), mycelial conidiophores (Fig. 6), and dichotomously branching hyphae (Fig. 7) was revealed on periodic acid Schiff (PAS) staining in $70 \%$ of cases while hyphae was common in all cases, which is pathognomonic in diagnosis by special staining. 


\section{Aspergillosis at Dhaka Zoo}

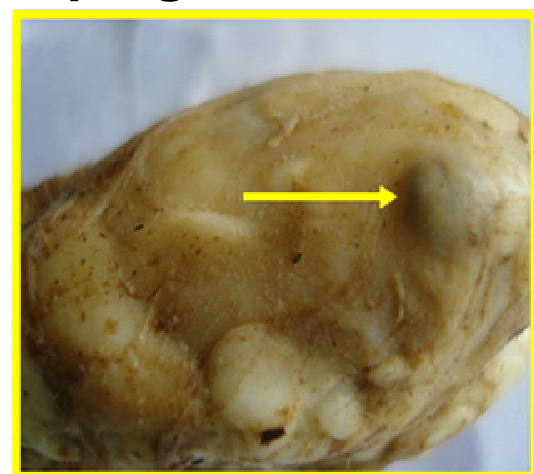

Fig. 1: Lymphnode of Samber deer, presence of large caseous nodules blackish in color

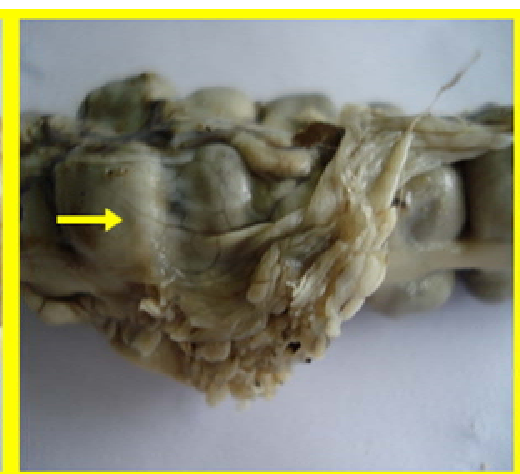

Fig. 2: Intestine of Common langur, numerous minute to large nodules greenish in color

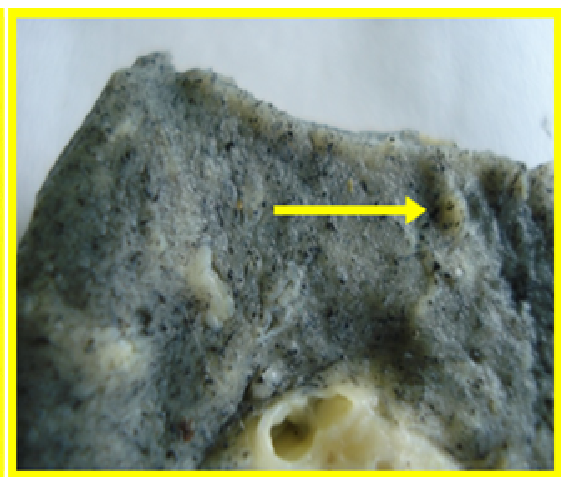

Fig. 3: Lung of Striped hyena, presence of tiny nodules yellow white in color
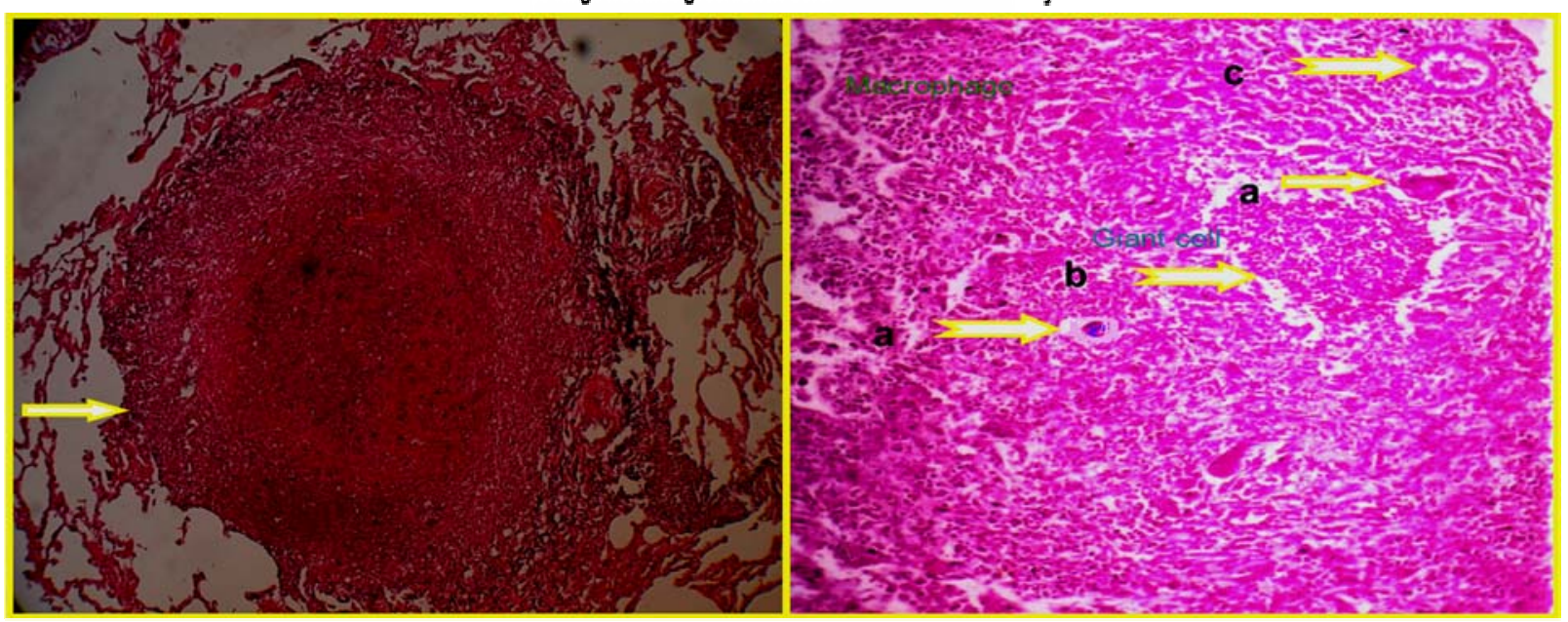

Fig. 4: Lung of rhesus macaque showing larger granuloma, multifocal, severe, granulomatous pneumonia, H\&E, $\times 200$.

Fig. 5: Spleen of rhesus macaque showing formation of giant cells (a), fungal spores (b), radiating club (c) severe, granulomatous mycotic spleenitis, H\&E, $\times 82.5$.
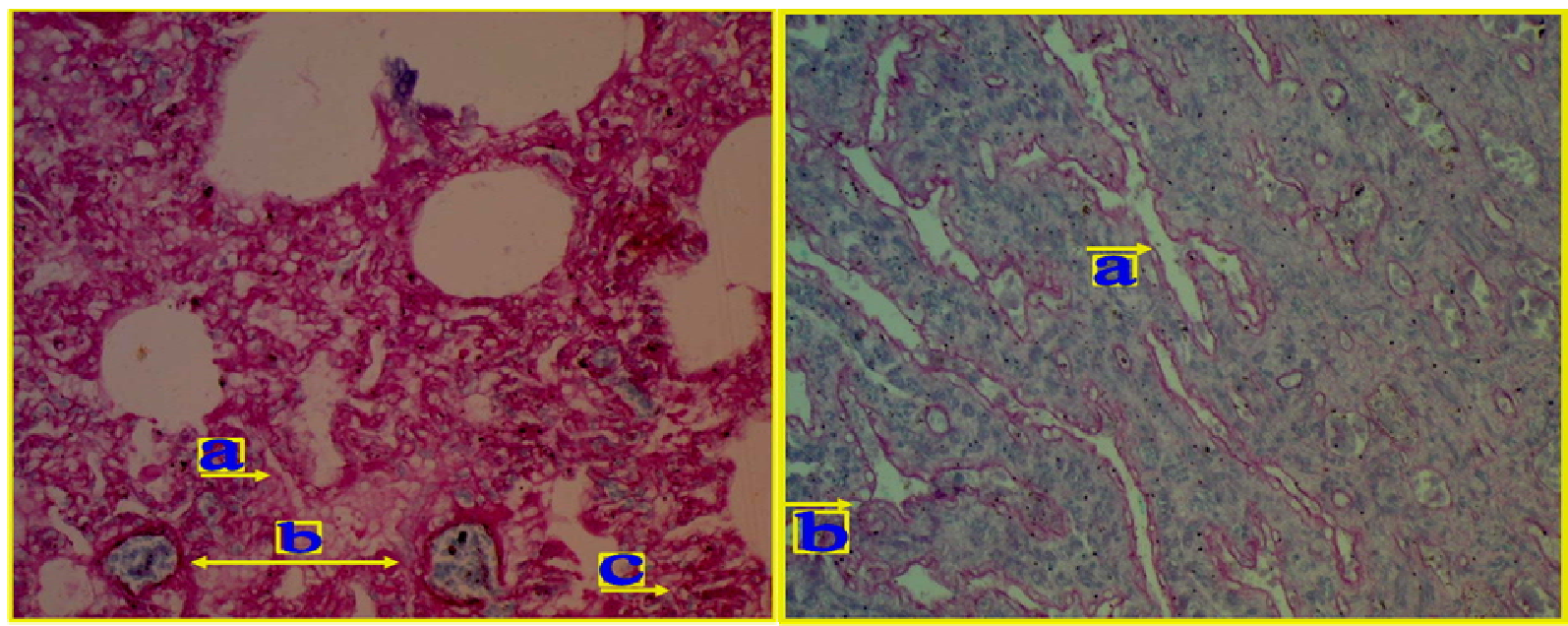

Fig. 6: Lung of samber deer, presence of dichotomously Fig. 7: Intestine of Nilgai, numerous dichotomously branching septate hyphae (a) and mycelial conidiophore branching septate hyphae (a), mycelial cinidiophore (b), (b), spore (c), chronic mycotic bronchopneumonia with diffuse, severe, mycotic enteritis, Aspergillosis, PAS, $\times 400$. bronchiectasis, Aspergillosis, PAS, $\times 200$. 
Present study revealed complex form of Aspergillosis concurrent with other granulomatous diseases in eight cases. It was noticed complexed with blastomycosis, coccidioidomycosis and cryptococcosis in each of single case along with concomitant with blastomycosis and coccidioidomycosis while four aspergillosis cases were noticed and complexed with coccidioidomycosis, cryptococcosis and histoplasmosis. Five cases of aspergillosis was identified complexed with mycobacteriosis. Without unnoticeable degree of variation, clinical history noted was almost similar to other works, nonetheless, it was difficult to differentiate among other granulomatous infections. Frequently observed clinical signs of coughing and anorexia were prominent; gradual emaciation followed by death while coughing and anorexia in cases of Aspergillosis was comparable to many other studies. Yellow white tiny to large nodules on visceral organs at necropsy were typical to findings (Breshears et al., 2007; Mackinnon et al., 2000; Muntz, 1999; Monteros et al., 1999; Jones et al., 1997) but confusing whether it predisposed by fungus or other long standing infectious agent (Lisle et al., 2002). Infrequently, non-correspondance to granulomatous reaction with nodular lesions was also observed.

Most literature suggests the presence of foreign body giant cells in case of mycosis with the exception of findings by Jones et al. (1997) that suggests formation of both Langhan's and foreign body giant cells in case of blastomycosis while present investigation showed presence of both type of giant cells in case of aspergillosis with huge number of Langhan's type giant cells than foreign body.

Aspergillosis in rhesus macaque, Samber deer and horse was reported, those are similar with the present study but data were not available incase of striped hyena, gayal, nilgai, beisa oryx, water buck and greater kudu to compare this study. PAS in this study showed characteristic dichotomously branching septate hyphae, occasional radiating clubs and mycelial conidiophore. Findings of aspergillosis were similar with other works (Breshears et al., 2007; Mackinnon et al., 2000; Muntz, 1999; Monteros et al., 1999; Jones et al., 1997). Dhaka zoo animal might possess the infection from animal importation that were infected, dirty damp environment, contaminated or damp foods, and malnourishment or due to compromised immunity. This disease in Zoo animals can be confused with some other fungal diseases in particular blastomycosis, cryptococcosis, histoplasmosis, coccidioidomycosis, and mycobacteriosis by both their clinical manifestation and necropsy findings.

However, further studies should be focused on typing and molecular characterization of aspergillus organism. Aspergillus organism possesses serious public health consequences and therefore, prevention and control of these diseases in Dhaka zoo should brought under notice to ensure health safety standard.

\section{Acknowledgements}

We are thankful to the Department of Surgery and Obstetrics for photography.

\section{References}

Ahasan, S.A. and Azam, S.U. 2007. Contribution of Zoological Gardens towards conservation of Wildlife and Biodiversity Bangladesh perspective. Zoos' print Magazine, 22: 13-16.

Breshears, M.A., Holbrook, T.C., Haak, C.E. and York, P.A. 2007. Pulmonary Aspergillosis and Ischemic Distal Limb Necrosis Associated with Enteric Salmonellosis in a Foal, Vet Pathol. 44:215-217.

Dollinger, P. 2006. Marketing the conservation role of zoos' and aquariums, Zoos' Print Magazine. 21:17.

Gary, W., Thomas, D.L., Kurt, V. and Peter, B. 2003. Mycobacterial diseases in wildlife; procedings of $10^{\text {th }}$ conference on wildlife damage management, Internet center for USDA National Wildlife Research Center, University of Nebraska, Lincon, Staff Publication (K.A. Fagerstone, G.W. Witmer, Eds): 310-15 pp.

Jones, T.C., Hunt, R.D. and King, N.W. 1997. Diseases caused by fungi in Veterinary Pathology, $6^{\text {th }}$ edn., Williams and Wilkins. 8:506-522pp.

Lisle, D.G.W., Bengis, R.G., Schmitt, S.M. and O'Brien, D.J. 2002. Tuberculosis in freeranging wildlife: detection, diagnosis and management. Revue Scientifique at Technique des Office International des Epizooties. 21:317-334.

Luna, L.G. 1968. Manual of histologic staining methods of the Armed Forces Institute of Pathology. New York, 3rd Ed. (258 pages), mcgraw-Hill Book Co., New York, pp. 32-46. 
Mackinnon, J.E., Conti-Diaz, Ismael, A., Gezuele, E. and Civila, E. 2000. Relationships between wild entourages and mycoses, especially south american blastomycosis, Current Opinion in Microbiology. 3: 339-343.

Mallory, F.B. 1968. Pathological Technique, New York, Hafner Publishing Co. 275. AFIP modification.

McManus, J.F.A. 1948. Stain technique (AIFP modification) 23:99 - 108.

Monteros, A. E. 1999. Nasal zygomycosis and pulmonary aspergillosis in an American bison, Journal of Wildlife Diseases. 35:790795.

Muntz, F.H.A. 1999. Oxalate-producing Pulmonary Aspergillosis in an Alpaca, Vet Pathol. 36:631-632.

OIE (Office International des Epizootic). 2000. Zoonoses transmissible from non-human primates. Chapter 2.10.1. In International animal health code, 9th Ed. OIF, Paris. 285-290pp.

O'Reilly, L.M. and Daborn, C.J. 1995. The epidemiology of Mycobacterium bovis infections in animals and man. A review, Tubercle and Lung Disease, Supplement 1, August, 76: 1-46.

Peter J.S.O. 2005. Building a future for wildlife, The World Zoo and Aquarium Conservation Strategy eds. By World Association Zoos and Aquqriums (WAZA), 7-10pp.

Rahman, A.Z. and Ahasan, S.A. 2006. Spotted deer (Cervus axis) herd in Dhaka Zoo: case report. Zoos' Print, 21.

Rahman, A.Z. and Ahasan, S.A. 2007. Mortality in Dhaka Zoo due to microbial agents. Bangladesh J Microbiol, 24:154-156.

Renquist, D.M. and Whitney, R.A. 1978. Tuberculosis in non-human primates - an overview. In Proc. Symposium on mycobacterial infections in zoo animals (R.J. Montali, ed.), 6-8 October 1976, Front Royal, Virginia. Smithsonian Institution Press, Washington, DC. 9-16.

Salem, D.J. and Rowan, A.N. 2001. The State of the Animals: Humane Society Press, Gaithersburg, Maryland, Chapter 9: Hancocks, David, "Is There a Place in the World For Zoos?", 137-144; Farinato, Richard, "Another View of Zoos". 145147.

Tribe, A. 2004. "Zoo Tourism", in Wildlife Tourism: Impacts, management and planning. Higginbottom, Karen (ed.), Common Ground Publishing, Altona, Victoria. 35-56.

World Association Zoos and Aquqriums (WAZA). 2003. Code of ethics and animal welfare, proceedings of the $58^{\text {th }}$ annual meeting. San Joe's, WAZA executive Office, Berne. 139-142. www.waza.org/en/site/conservation/code-of-ethics-animal-welfare; accessed 26 November 2010. 